\title{
Urinary Periostin as an Early Predictor of Renal Injury in Diabetic Kidney Disease: A Tertiary Centre Experience
}

\author{
Munna Lal Patel', Rekha Sachan², Durgesh Pushkar', Shyam Chand Chaudhary' ${ }^{1}$, Kamlesh Kumar Gupta'
}

\section{Munna Lal Patel ${ }^{1}$, Rekha Sachan ${ }^{2}$, Durgesh Pushkar', Shyam Chand Chaudhary', Kamlesh Kumar Gupta'}

'Department of Medicine, King Georges Medical University, Lucknow, Uttar Pradesh, INDIA.

${ }^{2}$ Department of Obstetrics and Gynaecology, King George's Medical University, Lucknow, Uttar Pradesh, INDIA.

\section{Correspondence}

Dr. Munna Lal Patel,

Additional Professor, Department of Medicine, King George's Medical University, Lucknow, Uttar Pradesh, INDIA.

Mobile no: +919839007000

Email: patel.ml66@gmail.com

History

- Submission Date: 22-04-2018

- Revised Date: 12-09-2018

- Accepted Date: 31-10-2019

DOI : 10.5530/ijmedph.2019.3.23

Article Available online

http://www.ijmedph.org/v9/i3

\section{Copyright}

(c) 2019 Phcog.Net. This is an openaccess article distributed under the terms of the Creative Commons Attribution 4.0 International license.

\begin{abstract}
Introduction: Early and accurate detection of onset of diabetic nephropathy is crucial for a timely intervention and for improving outcomes in the management of diabetic nephropathy. There is a need of new biomarkers to predict the onset of nephropathy earlier than the conventional markers. The aim of this study was to measure the association of urinary periostin level in type 2 subjects and healthy controls and to evaluate its predictive value to diagnose renal injury. Methods: This was a cross sectional study in which urine samples were collected from 50 healthy controls and 360 type 2 diabetic patients. The diabetic cases were further grouped as; normoalbuminuria $(n=95)$, microalbuminuria $(n=130)$ and macroalbuminuria $(n=135)$. The urinary levels of periostin was measured by using an enzyme-linked immunosorbent assay kit. Observations: Urinary periostin levels were found to be significantly elevated in all the diabetic patients compared to the controls. In multivariate regression analysis increased urine periostin level significantly correlated with aging, high albuminuria and decline of eGFR. For differentiating normalbuminuria, microalbumiuria and macroalbuminuria the best selected cut-off values with optimum sensitivity and specificity were $>2.11,2.74$ and 7.81 respectively with predicted sensitivity and specificity of $88 \%$ and $100 \%, 98.1 \%$ and $66.7 \%$ and $100 \%$ and $69.9 \%$ respectively. Therefore, urinary periostin demonstrated moderate to high sensitivity and specificity for diagnosing diabetic nephropathy. Conclusion: Increased levels of urinary periostin significantly correlated with aging, high albuminuria, declinement of eGFR and can be detected in the urine of the patients with type 2 diabetes before the onset of significant albuminuria. Urinary periostin excretion may be used as an early biomarker of renal injury.

Key words: Urinary Periostin, Biomarker, Renal Injury, Diabetic Kidney Disease.
\end{abstract}

\section{INTRODUCTION}

Diabetic nephropathy is a common complication of diabetes and the leading cause of chronic kidney disease. Approximately $40 \%$ of persons with diabetes develop diabetic nephropathy, manifested as albuminuria and/or decreased glomerular filtration rate. Nephropathy in diabetic patients is characterized by functional as well as structural abnormalities. ${ }^{1}$ In addition to glomerular pathology, it also involves tubulointerstitial compartment causing the expansion of tubular basement membranes, tubular atrophy, interstitial fibrosis and arteriosclerosis. Tubulointerstitial damage is one of the important factors in the pathophysiology and progression of nephropathy. ${ }^{2}$ Moreover, it has been proposed that tubular injury could precede glomerular injury in diabetic nephropathy which may explain the early appearance of an increase in several urinary biomarker excretion compared with albumin. ${ }^{3}$ The grade of albuminuria is not a very sensitive marker of diabetic nephropathy. To screen for diabetic nephropathy in patients who are non albuminuric hence requires some other biomarker which can predict the onset of nephropathy. There are various serum and urinary biomarker to detect early onset nephropathy in diabetic subject but none is an absolute marker. Therefore, early detection

of nephropathy is of great importance to enable adequate treatment in these patients and improve their outcomes.

Periostinis an extracellular matrix protein of mesenchymal origin and serves as an adhesion molecule for pre-osteoblasts. Periostin is undetected in the normal renal tubules and is released when distal tubules are damaged, thus Periostin can be used as an early marker for renal injury. ${ }^{4}$ Very few study done on periostin as biomarker of renal injury, so we planned our study with aim to investigate the association of urinary periostin levels in normal healthy control and in diabetic nephropathy and to evaluate its predictive value to diagnose renal injury.

\section{MATERIALS AND METHODS}

This cross sectional study was carried out in the Department of Medicine, King George's Medical University, Lucknow, Uttar Pradesh, India over a period of one year from September 2015 to August 2016. After informed written consent and approval from Institutional Ethics Committee patients were enrolled in the study. Patients with type 2 diabetes mellitus and aged more than 18 years were included in the study. A to-

Cite this article : Patel ML, Sachan R, Pushkar DK, Chaudhary SC, Gupta KK. Urinary Periostin as an Early Predictor of Renal Injury in Diabetic Kidney Disease: A Tertiary Centre Experience. Int J Med Public Health. 2019;9(3):100-4. 
tal 360 cases and 50 normal healthy controls were enrolled in the study. Patients suffering from active urinary tract infection, nephropathy other than diabetes, neoplastic disorders, chronic liver disease, inflammatory disorders, pregnancy and subjects who were not willing to take part in the study were excluded from the study.

The Modification of Diet in Renal Disease formula was used to calculate the estimated Glomerular Filtration Rate (eGFR). Microalbuminuria (defined as urine albumin between 30 and $300 \mathrm{mg} / 24 \mathrm{hr}, 20-200 \mu \mathrm{g} /$ min on a timed sample, or spot urine albumin to creatinine ratio 30-300 $\mathrm{mg} / \mathrm{g}$ ) and albuminuria, also termed clinical albuminuria, macroalbuminuria and overt nephropathy $(>300 \mathrm{mg} / 24 \mathrm{hr},>200 \mu \mathrm{g} / \mathrm{min}$ on a timed sample, or spot urine albumin to creatinine [ACR] ratio $>300 \mathrm{mg} / \mathrm{g}$ ) The diagnosis of diabetic nephropathy as recommended by the American Diabetes Association guidelines is to measure albumin in a spot urine sample and estimated GFR. Presence of any abnormalities in either of the two is sufficient to detect diabetic kidney disease. ${ }^{5}$ NDRD [Non Diabetic Renal Disease] in diabetic patients defined as sudden onset of proteinuria, proteinuria in the absence of diabetic retinopathy, active urinary sediment, rapidly decreasing renal function and short duration of diabetes. ${ }^{6,7}$ After overnight fasting $5 \mathrm{ml}$ of venous sample was withdrawn with full aseptic precaution for routine hemogram, fasting and postprandial blood glucose, glycosylated haemoglobin (HBA1c), lipid profile, serum protein, serum albumin, blood urea and serum creatinine. An automated blood-cell analyser (BC-5380; Mindray, Shenzhen, People's Republic of China) was used for routine haematology testing and an automated clinical biochemistry analyser (Cobas C 311; Roche-Hitachi, Tokyo, Japan) was used for blood urea, creatinine, electrolytes, serum lipids, protein and albumin.

A clean, morning midstream $5 \mathrm{~mL}$ urine sample was collected from healthy subjects and patients with type 2 diabetes with various stages of nephropathy into a sterile test tube and centrifuged at 5,000 rpm for 15 min. The supernatant was transferred to an Eppendorf tube and stored at $-80^{\circ} \mathrm{C}$ until assay done for Periostin. Human Perisotin Elisa kit (Thermo scientific, Freidrick, MD, USA) was used for estimation of Periostin in the urine as per the manufacturer's protocol.

\section{Statistical analysis}

Results were expressed as means \pm SD and median for continuous variables or as a percentage in categorical variables. Statistical analysis was performed using SPSS, version 15. For continuous variables either the two-sample $t$ test or Mann-Whitney rank test was used. For multiple comparisons, ANOVA was used followed by the least significance difference test. Multiple regression was used to detect urine periostin and the relevancy of each parameter.

Finally, Receiver Operating Characteristics (ROC) analysis was used to calculate the Area Under the Curve (AUC) for periostin and to find the best cut-off values to identify diabetic Nephropathy. Results with $p<0.05$ were considered statistically significant.

\section{OBSERVATIONS AND RESULTS}

A total of 367 subjects with type 2 diabetes mellitus were enrolled in our study, out of which 7 patients were dropped out from the study, so analysis was performed on 360 patients. Simultaneously 50 normal healthy subjects were also enrolled who served as controls. These were further grouped as 95 (26.38\%) normoalbuminuric, 130(36.1\%) with microalbuminuria and remaining 135 (37.5\%) in macroalbuminuria group. Mean age of patients in control, normoalbuminuria, microalbuminuria and macroalbuminuria groups were $48.6 \pm 6.0,50.2 \pm 9.98,50.83 \pm 6.94$ and $52.94 \pm 4.96$ years respectively. Majority of patients in all the groups except those in microalbuminuria were males. However, the difference among groups was not significant statistically $(p=0.546)$. (Table 1 )

Mean HBA1c and fasting blood glucose levels in all diabetic groups were controlled $(p<0.001)$. It was also observed that significant intergroup difference was present in the mean systolic blood pressure values $(p<0.05)$. An increasing trend of systolic blood pressure was observed with the severity of nephropathy. (Table 2)

In our study the mean triglyceride values in all diabetic patients were raised $(p<0.003)$. It was also found that low HDL type of dyslipidemia was present in all the diabetic groups $(p<0.001)$. Urinary Albumin Creatinine Ratio (UACR) was significantly elevated in patients with advanced diabetic nephropathy compared with type 2 diabetes with normoalbuminuria and healthy controls. However no significant difference of UACR was observed between normoalbuminuria and control groups (Table 2, Figure 1)

To evaluate the importance of periostin as a marker of kidney disease progression, we performed regression analysis with the diabetic nephropathy severity as dependent variables. In univariate analysis, urine periostin levels were positively correlated with age, fasting plasma glucose, HBA1C, total cholesterol and LDL, but negatively correlated with

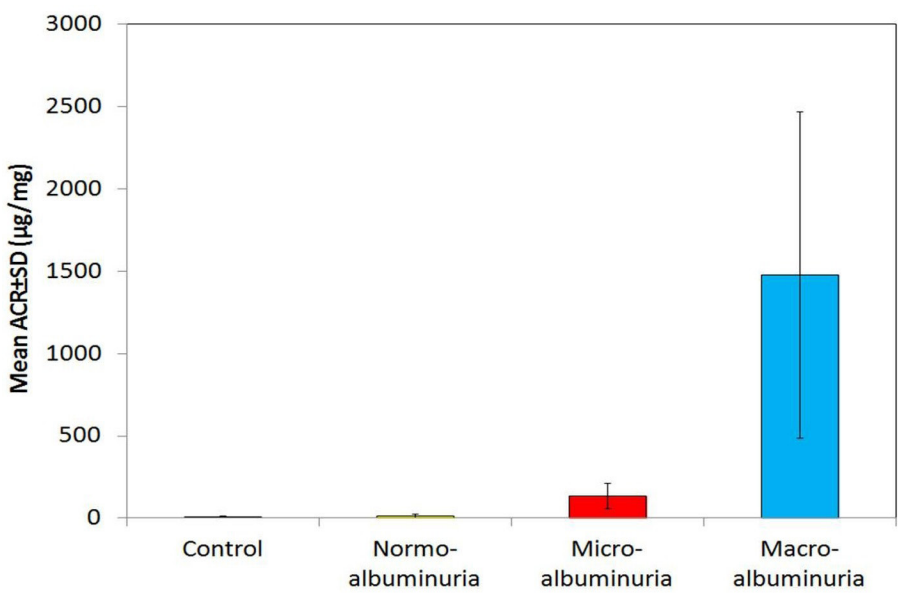

Figure 1: Bar diagram showing grade of UACR in different groups.

\begin{tabular}{|c|c|c|c|c|c|c|}
\hline SN & Parameter & $\begin{array}{l}\text { Control } \\
(n=50)\end{array}$ & $\begin{array}{l}\text { Normo- } \\
\text { albuminuria } \\
(95)\end{array}$ & $\begin{array}{l}\text { Micro- } \\
\text { albuminuria } \\
(n=130)\end{array}$ & $\begin{array}{l}\text { Macro- } \\
\text { albuminuria } \\
(n=135)\end{array}$ & $\begin{array}{c}\text { Statistical } \\
\text { significance }\end{array}$ \\
\hline 1. & $\begin{array}{l}\text { Mean Age } \pm \text { SD } \\
\text { (Range) in yrs }\end{array}$ & $\begin{array}{c}48.6 \pm 6.0 \\
(35-55)\end{array}$ & $50.2 \pm 9.98(32-65)$ & $\begin{array}{c}50.83 \pm 6.94(34- \\
65)\end{array}$ & $\begin{array}{c}52.94 \pm 4.96(45- \\
65)\end{array}$ & $\begin{array}{l}\mathrm{F}=9.613 \\
p<0.001\end{array}$ \\
\hline \multirow[t]{3}{*}{2.} & Gender & & & & & \\
\hline & Male & $8(57.1 \%)$ & $10(43.5 \%)$ & $22(61.1 \%)$ & $10(62.5 \%)$ & \multirow{2}{*}{$\begin{array}{c}\chi^{2}=2.128 \\
p=0.546 \text { (NS) }\end{array}$} \\
\hline & Female & $6(42.9 \%)$ & $13(56.5 \%)$ & $14(38.9 \%)$ & $6(37.5 \%)$ & \\
\hline
\end{tabular}




\begin{tabular}{|c|c|c|c|c|c|c|c|c|c|c|}
\hline Parameter & \multicolumn{2}{|c|}{ Control $(n=50)$} & \multicolumn{2}{|c|}{$\begin{array}{c}\text { Normo-albuminuria } \\
\text { (95) }\end{array}$} & \multicolumn{2}{|c|}{$\begin{array}{l}\text { Micro-albuminuria } \\
\quad(n=130)\end{array}$} & \multicolumn{2}{|c|}{$\begin{array}{l}\text { Macro-albuminuria } \\
\qquad(n=135)\end{array}$} & \multicolumn{2}{|c|}{$\begin{array}{c}\text { Statistical } \\
\text { significance }\end{array}$} \\
\hline $\operatorname{ACR}(u g / m g)$ & 8.5 & 5.0 & 15.1 & 9.6 & 136.6 & 77.9 & 1478 & 991 & 49.59 & $<0.001$ \\
\hline TC (mg/dl) & 158.1 & 23.7 & 158.1 & 34.9 & 150.6 & 45.1 & 159.4 & 48.4 & 0.272 & 0.845 \\
\hline $\mathrm{LDL}(\mathrm{mg} / \mathrm{dl})$ & 88.6 & 32.5 & 100.4 & 35.3 & 87.7 & 41.0 & 98.1 & 41.2 & 0.67 & 0.574 \\
\hline $\operatorname{eGFR}\left(\mathrm{ml} / \mathrm{min} / 1.73 \mathrm{~m}^{2}\right)$ & 132.9 & 12.2 & 99.7 & 24.0 & 88.6 & 15.3 & 74.1 & 13.4 & 32.33 & $<0.001$ \\
\hline HBAlc (\%) & 4.96 & 0.34 & 6.70 & 1.99 & 6.64 & 2.80 & 6.36 & 1.79 & 21.36 & $<0.001$ \\
\hline FBS (mg/dl) & 86.43 & 8.64 & 100.57 & 21.49 & 110.00 & 27.04 & 104.88 & 35.42 & 11.78 & $<0.001$ \\
\hline
\end{tabular}

\begin{tabular}{|c|c|c|c|c|c|}
\hline & \multicolumn{2}{|c|}{ Univariate } & \multicolumn{3}{|c|}{ Multivariate } \\
\hline & $\mathbf{R}$ & $P$-value & Beta & $95 \% \mathrm{Cl}$ & $p$-value \\
\hline Age (Years) & 0.158 & 0.003 & 135.70 & 14.25 to 254.18 & 0.032 \\
\hline FBS (mg/dl) & 0.283 & $<0.001$ & 5.60 & -16.50 to 27.87 & 0.662 \\
\hline HbAlc (\%) & 0.116 & 0.046 & 70.40 & -455.15 to 596.92 & 0.859 \\
\hline $\mathrm{LDL}(\mathrm{mg} / \mathrm{dl})$ & -0.108 & 0.048 & -5.30 & -47.25 to 56.60 & 0.921 \\
\hline $\begin{array}{l}\mathrm{UACR}(\mathrm{ml} / \\
\mathrm{min})\end{array}$ & 0.560 & $<0.001$ & 16.92 & 11.52 to 24.20 & $<0.001$ \\
\hline $\begin{array}{l}\text { eGFR }(\mathrm{ml} / \\
\left.\min / 173 \mathrm{~m}^{2}\right)\end{array}$ & -0.083 & $<0.018$ & -82.64 & -32.35 to -132.92 & 0.002 \\
\hline
\end{tabular}

Independent variable in the multivariate linear regression model were chosen using a step wise regression analysis where all variables listed in univariate were included. FBS- Fasting blood sugar; LDL- Lower density cholesterol, HbAlcHemoglobin AK, UACR- Urinal albumin creatinine ratio, eGFR- estimated glomerular filteration rate

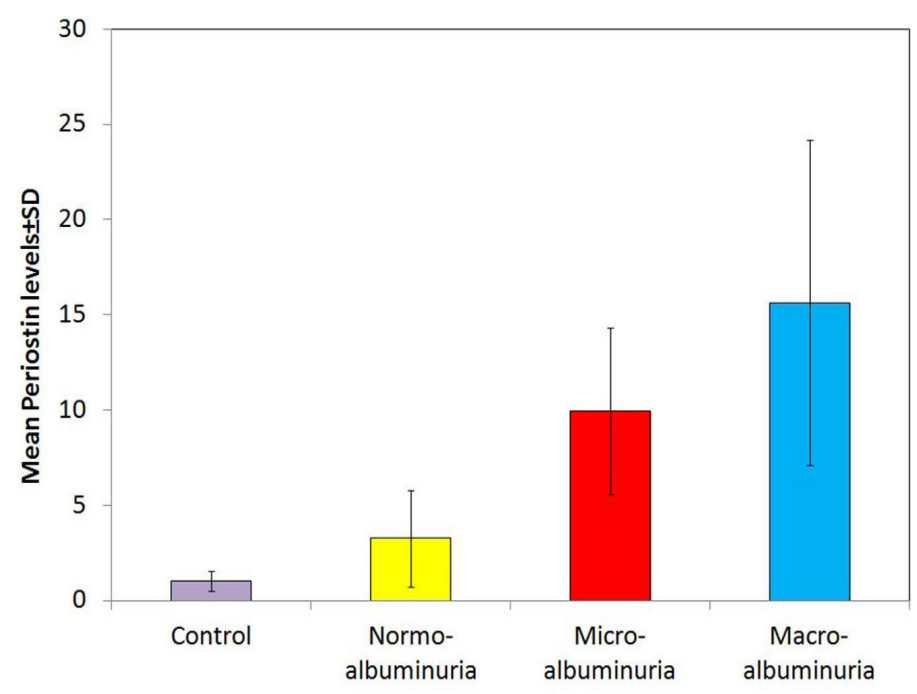

Figure 2: Mean periostin values $(\mathrm{ng} / \mathrm{ml})$ in various groups of patient.
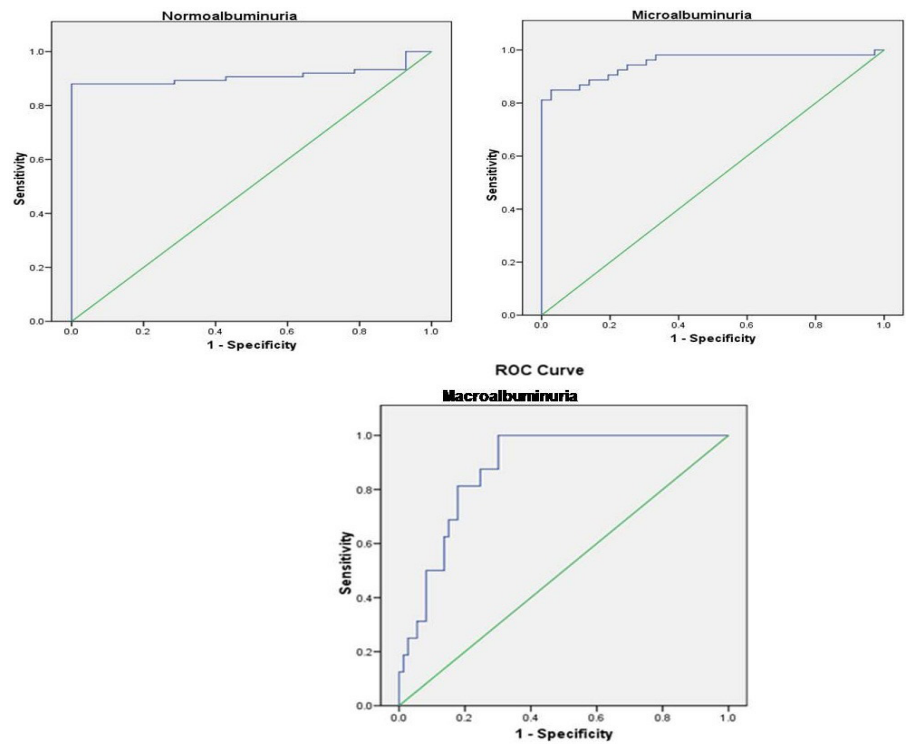

Figure 3: ROC curves showing sensitivity and specificity of periostin in various groups.

eGFR. After multiple regression analyses, Urinary periostin levels were positively correlated with albuminuria, advanced age and declinement of eGFR. In general, the increase in urine albumin and the decrease in eGFR represent the aggravated renal function in patients with diabetic nephropathy. These data are consistent with the hypothesis that urinary periostin levels were associated with exaggerated renal function in type 2 diabetes patients. (Table 3 )

In our study the urinary levels of periostin in normo-albuminuric patients was higher as compared to the controls and there was a progressive increase in urinary periostin levels with the progression of stage of nephropathy. It was found that the mean periostin levels showed a significant increasing trend from control to macroalbuminuria $(p<0.001)$. The appearance of urine periostin in type 2 diabetes patients but not in healthy controls underscores its value as a potential biomarker for kidney injury in albuminuric and nonalbuminuric type 2 diabetes. (Table 4, Figure 2)

The AUC for differentiating normoalbuminuria from controls was 0.910 , microalbuminuria from normoalbuminuria was 0.951 and macroalbu- 


\begin{tabular}{|c|c|c|c|c|c|c|}
\hline \multirow[t]{2}{*}{ Group } & \multirow{2}{*}{$\begin{array}{c}\text { Cut-off } \\
\text { value }(\geq)\end{array}$} & \multirow[t]{2}{*}{ Sensitivity (\%) } & \multirow[t]{2}{*}{ Specificity (\%) } & \multirow[t]{2}{*}{ Area } & \multicolumn{2}{|c|}{$95 \% \mathrm{Cl}$} \\
\hline & & & & & Lower & Upper \\
\hline \multirow[t]{3}{*}{ Normoalbuminuria } & 2.11 & 88.0 & 100 & 0.910 & 0.848 & 0.971 \\
\hline & 0.89 & 90.7 & 57.1 & & & \\
\hline & 0.67 & 92.0 & 42.9 & & & \\
\hline \multirow[t]{3}{*}{ Microalbuminuria } & 2.74 & 98.1 & 66.7 & 0.951 & 0.907 & 0.996 \\
\hline & 4.31 & 94.3 & 72.2 & & & \\
\hline & 5.08 & 90.6 & 80.6 & & & \\
\hline \multirow[t]{3}{*}{ Macroalbuminuria } & 7.81 & 100 & 69.9 & 0.877 & 0.805 & 0.949 \\
\hline & 9.40 & 87.5 & 75.3 & & & \\
\hline & 10.36 & 81.3 & 82.2 & & & \\
\hline
\end{tabular}

minuria from microalbuminuria was 0.877 . For differentiating normoalbuminuria, microalbumiuria and macroalbuminuria the best selected cut-off values with optimum sensitivity, specificity were $\geq 2.11,2.74$ and 7.81 respectively with predicted sensitivity and specificity of $88 \%$ and $100 \%, 98.1 \%$ and $66.7 \%$ and $100 \%$ and $69.9 \%$ respectively. At above cutoff value predicted sensitivity and specificity of $88 \%$ for discrimination between normoalbuminuria and control. To discriminate between normoalbuminuria and microalbuminuria sensitivity and specificity was $98.1 \%$ and $66.7 \%$. (Table 4, Figure 3)

\section{DISCUSSION}

Diabetic nephropathy is a serious and late complication of diabetes mellitus thus it is necessary to diagnose and treat comprehensively because of its significant impact on occurrence of cardiovascular events as well as death due to renal failure. ${ }^{8}$ Diabetic nephropathy is the most common cause of chronic kidney disease in almost every population including India. ${ }^{9}$ Currently used marker for screening of diabetic nephropathy is the measurement of albumin excretion in urine and eGFR as recommended by the ADA. ${ }^{5}$ Although UACR has a good sensitivity but it has its own limitations as well. Albuminuria may be increased in episodic hyperglycaemia, high blood pressure, high-protein diet, exercise, fever, urinary tract infection and congestive heart failure, thus screening in people affected with such conditions can lead to inaccurate values. ${ }^{10}$ Recently there is a growing concept of diabetic tubulopathy in the pathogenesis of diabetic kidney disease. Tubular damage is not only a marker of kidney damage in diabetic nephropathy but also a strong predictor of progression of renal damage than the glomerular injury. ${ }^{11}$ Tubular injury markers are thus being studied and they have shown promising results in diagnosing diabetic nephropathy in the earlier stages. The occurrence of tubular damage in the early stages of diabetic nephropathy has been reported in various studies done on urinary biomarkers in diabetic patients. ${ }^{12-16}$ In our study we tested urinary periostin as a biomarker to diagnose renal injury in type 2 diabetic subjects. Periostin expression in kidney and its detection in urine during renal injury has been demonstrated in very few studies. Periostin acts by promoting cellular dedifferentiation, extracellular matrix deposition and increased TGF-beta expression. ${ }^{17}$ Periostin helps in renal tissue remodelling and regeneration in normal as well as in diseased state. ${ }^{18}$ In univariate analysis, urine periostin levels were positively correlated with age, fasting plasma glucose, $\mathrm{HbA1c}$ and LDL but negatively correlated with eGFR. After multiple regression analyses, urine periostin levels were positively correlated with albuminuria, advanced age and declinement of eGFR. Our study recapitulates the findings of study done by Satirapoj B et al. Where urine periostin levels were positively correlated with increased albuminuria, advanced age and decline of estimated GFR.${ }^{19}$ Periostin is undetected in the normal renal tubules and is released when distal tubules are damaged. Our study demonstrated that mean urinary periostin levels were significantly higher in type 2 diabetics than the controls. Also urinary periostin excretion positively correlated with disease severity in subjects with diabetic nephropathy. Our study is in accordance to study done by Satirapoj B et al. where urinary periostin levels were significantly elevated in the patients of the normoalbuminuria, microalbuminuria and macroalbuminuria compared with healthy controls. ${ }^{19}$ In our study for differentiating normolbuminuria, microalbumiuria and macroalbuminuria the best selected cut-off values were $\geq 2.11,2.74$ and 7.81 respectively with predicted sensitivity and specificity of $88 \%$ and $100 \%, 98.1 \%$ and $66.7 \%$ and $100 \%$ and $69.9 \%$ respectively. In study of Satirapoj B et al the cut-off levels of periostin were $1.01 \mathrm{ng} / \mathrm{mgCr}$ (sensitivity $80.7 \%$, specificity $43.3 \%$ ), $1.13 \mathrm{ng} / \mathrm{mgCr}$ (sensitivity $74.6 \%$, specificity $50.0 \%$ ), 1.39 $\mathrm{ng} / \mathrm{mgCr}$ (sensitivity $71.1 \%$, specificity $66.7 \%$ ) and $1.47 \mathrm{ng} / \mathrm{mgCr}$ (sensitivity $69.3 \%$, specificity $70.0 \%$ ) to distinguish normoalbuminuric type 2 diabetes from healthy controls. ${ }^{19}$ One of the remarkable finding in our study is that in normoalbuminuric diabetic at cut-off values of 2.11 , the sensitivity and specificity was $88 \%$ and $100 \%$. Therefore, urine periostin ELISA demonstrated moderate to high sensitivity and specificity for diagnosing diabetic nephropathy.

Our study findings were compatible to study done by Satirapoj B et al. Where urine periostin demonstrated high performance for the diagnosis of normoalbuminuric, microalbuminuric and macroalbuminuric type 2 diabetes (AUC 0.78 (95\%CI, 0.71 to 0.86 ), 0.99 (95\%CI, 0.98 to 1.00 ) and 0.95 (95\%CI, 0.91 to 0.98 ), respectively) as compared to our study value of AUC 0.910, 0.951 and 0.877 respectively. ${ }^{19}$ The limitations of our study was a small sample size. The results could be more conclusive and more propagative if carried out with a larger population. The study design being cross sectional, so we could not establish the role of periostin as a marker to monitor therapeutic response. Unfortunately we did not demonstrate periostin expression in renal tissue of diabetic nephropathy subjects. A prospective cohort study is hence needed to study the role of urinary perostin as a prognostic marker and as a marker for the monitoring of therapeutic response in diabetic patients.

\section{CONCLUSION}

In type 2 diabetic with normoalbuminuria, urinary periostin levels were significantly higher than in controls and urinary periostin excretion correlated with the severity of nephropathy in patients with type 2 diabetes. Elevated periostin levels in the urine may provide an opportunity for early diagnosis and advanced interventions in type 2 diabetes. 


\section{ACKNOWLEDGEMENT}

We acknowledge Dr. Wahid Ali Additional Professor, Department of Pathology, King George's Medical University, Lucknow for helping in estimation of periostin levels in urine. Dr. Arvind Misra, Professor, Department of Medicine, King George's Medical University, Lucknow for helping in recruitment of patients of diabetic nephropathy.

\section{CONFLICT OF INTEREST}

The authors declare no conflict of interest.

\section{ABBREVIATIONS}

eGFR: Estimated Glomerular filteration rate; MDRD: Modification of diet in renal disease; NDRD: Non diabetic renal disease; HbA1C: Glycosylated hemoglobin; UACR: Urine Albumin to creatinine ratio; AUC: Area under curve; ROC: Receiver Operating Characteristics; ADA: American Diabetic Association; LDL: Low density Lipoprotein.

\section{REFERENCES}

1. Satirapoj B. Nephropathy in diabetes. Adv Exp Med Biol. 2012;771:107-22.

2. Zhou J, Chen $X, X i e Y$, et al. A differential diagnostic model of diabetic nephropathy and non-diabetic renal diseases. Nephrol Dial Transplant. 2008;23(6):1940-5.

3. Soni SS, Gowrishankar S, Kishan AG, et al. Non diabetic renal disease in type 2 diabetes mellitus. Nephrology. 2006;11(6):533-7.

4. Satirapoj B, Wang Y, Chamberlin M, Dai T, LaPage J, Phillips L, et al. Periostin: Novel tissue and urinary biomarker of progressive renal injury induces a coordinated mesenchymal phenotype in tubular cells. Nephrology Dialysis Transplantation. 2011;27(7):2702-11.

5. American Diabetes Association. Nephropathy in diabetes (Position Statement). Diabetes Care. 2004;27(Suppl 1):S79-83.

6. Tone A, Shikata K, Matsuda M, et al. Clinical features of non-diabetic renal diseases in patients with type 2 diabetes. Diabetes Res Clin Pract. 2005;69(3):23742.
7. Huang F, Yang Q, Chen L, et al. Renal pathological change in patients with type 2 diabetes is not always diabetic nephropathy: A report of 52 cases. Clin Nephrol. 2007;67(5):293-7.

8. Afkarian M, Sachs MC, Kestenbaum B, Hirsch IB, Tuttle KR, Himmelfarb J, et al. Kidney Disease and Increased Mortality Risk in Type 2 Diabetes. Journal of the American Society of Nephrology. 2013;24(2):302-8.

9. Rajapurkar M, John G, Kirpalani A, Abraham G, Agarwal S, Almeida A, et al. What do we know about chronic kidney disease in India: First report of the Indian CKD registry?. BMC Nephrol. 2012;13(1):10.

10. Bachmann LM, Nilsson G, Bruns DE, et al. State of the art for measurement of urine albumin: comparison of routine measurement procedures to isotope dilution tandem mass spectrometry. Clin Chem. 2014;60(3):471-80.

11. Thomas MC, Burns WC, Cooper ME. Tubular changes in early diabetic nephropathy. Adv Chronic Kidney Dis. 2005;12(2):177-86.

12. DeCarvalho JA, Tatsch E, Hausen BS, et al. Urinary kidney injury molecule-1 and neutrophil gelatinase-associated lipocalin as indicators of tubular damage in normoalbuminuric patients with type 2 diabetes. Clinical Biochemistry. 2016;49(3):232-6.

13. El-Ashmawy NE, El-Zamarany EA, Khedr NF, Abd El-Fattah Al, Eltoukhy SA Kidney injury molecule-1 (Kim-1): an early biomarker for nephropathy in type II diabetic patients. International Journal of Diabetes in Developing Countries. 2015;35(S3):431-8.

14. Viswanathan V, Sivakumar S, Sekar V, et al. Clinical significance of urinary livertype fatty acid binding protein at various stages of nephropathy. Indian Journal of Nephrology. 2015;25(5):269-73

15. Shore N, Khurshid R, Saleem M. Alpha1 microglobulin: A marker for early detection of tubular disorders in diabetic nephropathy. Journal of Ayub Medical College. 2010;22(4):53-5.

16. Saif A, Soliman N. Urinary $\alpha 1$-microglobulin and albumin excretion in children and adolescents with type 1 diabetes. Journal of Diabetes. 2016;9(1):61-4.

17. Horiuchi K, Amizuka N, Takeshita S, Takamatsu H, Katsuura M, Ozawa H, et al. Identification and Characterization of a Novel Protein, Periostin, with Restricted Expression to Periosteum and Periodontal Ligament and Increased Expression by Transforming Growth Factor $\beta$. Journal of Bone and Mineral Research. 1999;14(7):1239-49

18. Sorocos, Katrina et al. Expression Patterns and Roles of Periostin during Kidney and Ureter Development. The Journal of Urology. 2011;186(4):1537-44.

19. Satirapoj B, Tassanasorn S, Charoenpitakchai M, Supasyndh O. Periostin as a Tissue and Urinary Biomarker of Renal Injury in Type 2 Diabetes Mellitus. PLoS One. 2015:10(4):e0124055. 